\title{
Screening the Most Effective Variables on Physical Properties of Folate-Targeted Dextran/Retinoic Acid Micelles by Taguchi Design
}

\author{
M. Nayebsadrian, ${ }^{1}$ J. Varshosaz, ${ }^{1}$ F. Hassanzadeh, ${ }^{2}$ \\ H. Sadeghi, ${ }^{3}$ M. Banitalebi, ${ }^{1}$ and M. Rostami ${ }^{2}$ \\ ${ }^{1}$ Department of Pharmaceutics, School of Pharmacy and Novel Drug Delivery Systems Research Center, \\ Isfahan University of Medical Sciences, Isfahan 81745-359, Iran \\ ${ }^{2}$ Department of Medicinal Chemistry, School of Pharmacy, Isfahan University of Medical Sciences, Isfahan 81745-359, Iran \\ ${ }^{3}$ Department of Biotechnology, School of Pharmacy, Isfahan University of Medical Sciences, Isfahan 81745-359, Iran
}

Correspondence should be addressed to J. Varshosaz, varshosaz@pharm.mui.ac.ir

Received 18 August 2012; Accepted 12 November 2012

Academic Editor: Lian Gao

Copyright (C) 2012 M. Nayebsadrian et al. This is an open access article distributed under the Creative Commons Attribution License, which permits unrestricted use, distribution, and reproduction in any medium, provided the original work is properly cited.

Amphiphilic copolymers with self-assembling properties produce micelles in aqueous solutions and are made of two hydrophilic and hydrophobic segments. The objective of this study was optimization of the production of folate-conjugated dextran/retinoic acid (DEX/RA) micelles of doxorubicin. Micelles were prepared by direct dissolution method, and different effective parameters on their production were studied by a Taguchi design. The studied variables included CMC of the copolymer, polymer and drug contents, DEX Mw, stirring time, and rate and temperature. The effects of variables on responses of particle size, polydispersity index, zeta potential, drug loading, and release efficiency were studied. The most effective factors on particle size were DEX Mw, $\mathrm{CMC}$ of the copolymer, and stirring rate. Zeta potential and drug loading were more affected by the polymer content, DEX Mw, stirring time, and drug concentration. Stirring time and rate and also temperature of water were more effective variables on drug release rate. The optimum processing situation for micelles formation was obtained by copolymers with $\mathrm{CMC}<17 \mu \mathrm{g} / \mathrm{mL}$ and prepared from $20.1 \mathrm{mg}$ copolymers of DEX Mw of 10000 and drug concentration of $15.9 \%$. The optimized temperature for micelles formation was $40.7^{\circ} \mathrm{C}$, and stirring time was $1 \mathrm{hr}$ at a rate of $560 \mathrm{rpm}$.

\section{Introduction}

Amphiphilic copolymers with self-assembling properties produce micelles in aqueous solutions and are made of two hydrophilic and hydrophobic segments, with a large solubility difference. The hydrophobic part makes inner core of micelles that encapsulates drug, and the hydrophilic part makes outer shell that is exposed to the aqueous environment and protects micelle to be recognized by the reticuloendothelial system (RES) in vivo [1-3].

Polymeric micelles have been widely used as novel anticancer drug delivery systems over past decades to reduce drug degradation and loss, to decrease side effects and multiple drug resistance (MDR), and to increase drug bioavailability and the drug accumulation in the required site of the body. In comparison with other drug delivery systems, polymeric micelles have some advantages such as high stability, low critical micelle concentration (CMC), biodegradability, small particle size, high loading capacity, and prolonged circulation $[2,4]$.

For producing suitable polymeric micelles as drug delivery systems, development of new materials or improvement of existing materials has been tested over past years. Natural polymeric hydrogels (dextrans, albumin, chitosan, etc.) have been evaluated for this purpose in large number of studies [5]. Dextrans have been widely used because of their suitable properties such as nontoxicity, hydrophilic properties, nonimmunogenicity, stability, variety of molecular weights, low cost, and high ability of conjugation with other molecules via their large number of hydroxyl groups. They have been used 
as plasma volume expanders for many years. The structure of dextran consists of many glucose units with linear $\alpha$ 1,6 -glucosidic linkage and in some parts is branched via 1,3-glucosidic linkage. Dextrans have the ability to prolong circulation of the drug and thus enhance its therapeutic effects [6].

Dextrans should be conjugated with hydrophobic molecules to induce amphiphilic character and to produce polymeric micelles [5, 6]. In this study all-trans retinoic acid (RA) was chosen because of its anticancer effects against various cancers like acute myelogenous leukemia (AML), due to its ability to control differentiation, proliferation, and apoptosis of the cells, and it has the ability to form micelles with low CMC due to its poor water solubility $[7,8]$. RA is an active metabolite of retinol (vitamin A) in the biological metabolic pathway and used in chemotherapy and stem cell engineering due to its role in the control of cell differentiation [9]. Furthermore, its long chain of carbon atoms and carboxylate group makes it compatible for reacting with dextran and forming polymeric micelles in water. In recent studies, it has been discovered that RA upregulates folate receptor $\beta$ in KG1 cells [10].

Although polymeric micelles prolong circulation time and half life of drugs, a limitation of their application is insufficient uptake at tumour site and nonspecific delivery which causes side effects on normal tissues. One strategy to target polymeric micelles to cancerous cells is binding of a unique molecule to their surface. Recently, folic acid (folate) has attracted much attention as a targeting molecule due to overexpression of its receptors in many cancers like AML, ovarian cancer, nasopharyngeal, myeloid, and so forth [11].

To date many anticancer drugs have been tested for treating AML. Doxorubicin (Dox) is one of the most useful antibiotics against AML and some other cancers, but its use is limited by its severe side effects such as myelosuppression and cardiotoxicity $[12,13]$.

Development of folate- (FA-) grafted DEX/RA polymeric micelles have been reported previously [14] for specific delivery of Dox to FA receptor (+) KG-1 cells to increase its cytotoxicity and decrease its side effects. The objective of the present study was optimizing the factors affecting the production of FA-DEX/RA micelles of Dox.

\section{Materials and Methods}

2.1. Materials. Dextran Mw of 6000 and 10000 and dialysis tubing (cut-off $12 \mathrm{kDa}$ ) were purchased from Sigma (USA). Retinoic acid was purchased from Solmag Chemical Company (Italy); doxorubicin $\mathrm{HCl}$ (Hangzhou ICH Biopharm Co., Ltd., Zhejiang, China) and folic acid were from Merck Chemical Company (Germany).

2.2. Preparation of Micelles. The folate-conjugated DEX/RA copolymer was prepared as reported earlier [14] using carbonyldiimidazole and dimethylaminopyridine then Doxloaded micelles were prepared by direct dissolution method using the synthesized copolymer. For this method Dox and the copolymer were directly dissolved in distilled water. Loading of the drug into the micelles was done by stirring, heating, and then sonicating the mixture [15]. The CMC of different studied copolymers was determined as reported previously [14] using pyrene as a fluorescent probe. The micelles with CMC values less than $17 \mu \mathrm{g} / \mathrm{mL}$ were prepared by $\mathrm{D}_{10} \mathrm{R}_{15} \mathrm{~F}_{7}$ or $\mathrm{D}_{6} \mathrm{R}_{12} \mathrm{~F}_{4.2}$, and those with CMC values greater than $17 \mu \mathrm{g} / \mathrm{mL}$ were prepared by $\mathrm{D}_{10} \mathrm{R}_{10} \mathrm{~F}_{7}$ or $\mathrm{D}_{6} \mathrm{R}_{9} \mathrm{~F}_{4.2}$ copolymers. In these formulation codes the first digit represents the molecular weight of DEX (according to $\mathrm{KDa}$ ), the second one stands for the feeding mole of RA per each mole of DEX, and the last one shows the feeding mole of the targeting moiety, that is, folic acid (FA). To optimize the production process of the micelles loaded with Dox $\mathrm{HCl}$, the effect of various parameters was evaluated by a Taguchi design.

2.3. Experimental Design and Analysis. To evaluate the effect of processing variables on the particle size, zeta potential, encapsulation efficiency (EE\%), drug release efficiency (RE\%), and screening the most effective ones, a Taguchi design was used. Every experimenter has to plan and conduct experiments to obtain enough and relevant data, so that he can infer the science behind the observed phenomenon. $\mathrm{He}$ can do so by trial-and-error approach or by design of experiments. Taguchi has developed a method based on "orthogonal array" experiments which gives much reduced "variance" for the experiment with "optimum settings" of control parameters. Thus the marriage of design of experiments with optimization of control parameters to obtain best results is achieved in the Taguchi method. "Orthogonal arrays" provide a set of well-balanced (minimum) experiments and Dr. Taguchi's Signal-to-Noise ratios $(\mathrm{S} / \mathrm{N})$, which are log functions of desired output, serve as objective functions for optimization and help in data analysis and prediction of optimum results [16].

Identifying controlling factors, qualifying the magnitude of effects, identifying the statistically significant effects are emphasized in this design. The optimum conditions are determined by the Taguchi's optimization method to yield a heightened performance with the lowest possible effect of the noise factor. The first step of this method is to select the factor/level combination to maximize the response. The second step is to find the condition for attaining optimal desirability [16].

Seven different variables including CMC of the micelles, the content of copolymeric micelles, dextran Mw, stirring time, temperature, stirring rate, and drug concentration were studied each in two levels. Table 1 shows the seven control factors selected in the optimization study. A standard orthogonal array $\mathrm{L}_{8}$ was used to examine this system. $\mathrm{L}$ and subscript 8 denote the Latin square and the number of the experimental runs, respectively. An overview of the formulations investigated is presented in Table 2.

A run involved the corresponding combination of levels to which the factors in the experiment were set. All experiments were done in triplicate. The effects of the proposed experiments on the responses were then analyzed by the Design Expert software to obtain independently the main effects of these factors, followed by the analysis of variance (ANOVA) to determine which factors were statistically 
TABle 1: Description and trial levels of studied factors in Taguchi's $\mathrm{L}_{8}$ orthogonal array experiment on preparation of doxorubicin loaded in micelles of folate-targeted DEX/RA.

\begin{tabular}{lcc}
\hline Studied variables & \multicolumn{2}{c}{ Levels } \\
& I & II \\
\hline CMC $(\mu \mathrm{g} / \mathrm{mL})$ & $>17(\mu \mathrm{g} / \mathrm{mL})$ & $<17(\mu \mathrm{g} / \mathrm{mL})$ \\
Polymer content $(\mathrm{mg} / 100 \mathrm{~mL})$ & 27 & 20 \\
Dextran Mw $(\mathrm{Da})$ & 6000 & 10000 \\
Stirring time $(\mathrm{h})$ & 1 & 0.5 \\
Temperature $\left({ }^{\circ} \mathrm{C}\right)$ & 50 & 40 \\
Stirring rate $(\mathrm{rpm})$ & 400 & 600 \\
Drug concentration $(\%)$ & 30 & 15 \\
\hline
\end{tabular}

significant. The optimum conditions were determined by the Taguchi's optimization method to yield a heightened performance.

2.4. Particle Size and Zeta Potential Measurements. The particle size and zeta potential of the folate-conjugated DEX/RA micelles were measured by a zeta sizer (ZetasizerZEN 3600 Malvern Instrument Ltd., Worchestershire, UK). All particle size measurements were performed in deionized water using a He-Ne laser beam at $658 \mathrm{~nm}$ with a scattering angle of $130^{\circ}$.

2.5. Determination of Drug Loading in the Micelles. Drug loading efficiency (LE\%) was determined by measuring the concentration of unencapsulated free drug in aqueous medium. For this purpose $400 \mu \mathrm{L}$ of the drug-loaded micelles was centrifuged at $10000 \mathrm{rpm}$ for $15 \mathrm{~min}$ in Eppendorf tubes (cut-off $10000 \mathrm{Da}$ ), and the concentration of free drug in the aqueous medium was measured by a UV-vis spectrophotometer (UV-mini-1240CE-Shimadzu, Japan) at $\lambda_{\max }=270 \mathrm{~nm}$ in distilled water. Unloaded micelles were used as control. Loading efficiency (LE\%) was calculated by the following equation:

$$
\begin{aligned}
& \text { Drug loading efficiency } \% \\
& \quad=\frac{\text { Drug }_{\text {total }}-\text { Drug }_{\text {supernatant }}}{\text { Drug }} \times 100 .
\end{aligned}
$$

2.6. In Vitro Release of Dox from Micelles. The in vitro release of Dox from micelles was monitored in phosphate buffer solution (PBS) $0.2 \mathrm{M}$ (pH 7.4) containing 2\% of Tween 20. Six $\mathrm{mL}$ of aqueous micellar dispersion of each formulation was placed in the dialysis membrane bags (cut-off $12000 \mathrm{Da}$ ) and the end-sealed dialysis bags were sunk fully in $15 \mathrm{~mL}$ of release medium at room temperature. At appropriate time intervals the concentration of Dox released in the medium was determined by UV-spectrophotometry method at $\lambda_{\max }=$ $499.4 \mathrm{~nm}$.

\section{Results and Discussion}

3.1. Particle Size. The results of physicochemical properties of different formulations of micelles loaded with Dox are shown in Table 3.

Analysis of the results of this table by Design Expert software using Taguchi design indicated that the most effective parameter on the particle size of the micelles is DEX molecular weight (Figure 1). Changing the Mw of DEX from level 1 to level 2 (Table 1); that is, increasing the $\mathrm{Mw}$ from 6000 to $10000 \mathrm{Da}$ decreased the particle size of micelles effectively. The $\mathrm{CMC}$ value and stirring rate have also rather high contribution on the particle size (Figure 1). The copolymers with CMC values less than $17 \mu \mathrm{g} / \mathrm{mL}$ (i.e., level 2 of CMC in Table 1) had greater hydrophobic block core, which caused by an increase in the graft ratio. With this increase in the polymer hydrophobicity, the micelles formed in a more tightly packed core which results in smaller particle size. As the stirring rate increased (i.e., changing from level 1 to level 2 of stirring rate in Table 1), the particle size of the micelles decreased.

Polydispersity index (PDI) of the prepared micelles was between 0.257 and 0.495 (Table 3 ) which indicates low diversity of the particle size. Considering that the polydispersity (PdI) is calculated from the square of the standard deviation/mean diameter, less value of polydispersity index indicates enhanced homogeneity of the nanomicelles [17]. The suitable PdI of 0.2 is also reported in many works on nanoparticulate drug delivery systems like in PLGA nanoparticles of paclitaxel designed for antitumor activity in BT-549 cells [17] and chitosan nanoparticles cross linked with TPP [18].

3.2. Zeta Potential of Micelles. Zeta potential of the prepared micelles changed between almost -1 and $-8 \mathrm{mV}$ (Table 3 ). The effects of different studied parameters on the zeta potential of the micelles are shown in Figure 2. As this figure shows the most effective factor on zeta potential of micelles is the content of polymer. Decreasing the content of polymer (i.e., change from level 1 to level 2) increased the absolute value of zeta potential. This may be due to increase of the ratio of negative charge of the polymer and consequently enhancement of the surface charge of the micelles.

Other effective variables on zeta potential were drug concentration, stirring time, and dextran Mw. Decreasing the drug concentration (changing from level 1 to level 2) increased, the absolute value of zeta potential to more negative values. The charge of blank micelles was negative while Dox hydrochloride has positive charge. When the drug concentration increased the absolute value of zeta potential decreased, which indicates there are ionic interactions between folate-conjugated DEX/RA micelles and Dox hydrochloride.

Changing the stirring time from level 2 to level 1 , that is, increasing the stirring time increased the absolute value of zeta potential probably due to the induction of surface charge in micellar nanoparticles.

Increasing the DEX Mw (i.e., from level 1 to level 2) increased the absolute value of zeta potential. As described 
TABLE 2: Composition of different formulations investigated in preparation of doxorubicin loaded in folate-targeted DEX/RA micelles of $\mathrm{L}_{8}$ orthogonal array using Taguchi design.

\begin{tabular}{|c|c|c|c|c|c|c|c|}
\hline Run & $\begin{array}{c}\mathrm{CMC} \\
(\mu \mathrm{g} / \mathrm{mL})\end{array}$ & $\begin{array}{l}\text { Polymer content } \\
(\mathrm{mg} / 100 \mathrm{~mL})\end{array}$ & DEX Mw & Stirring time $(\mathrm{h})$ & Temperature $\left({ }^{\circ} \mathrm{C}\right)$ & $\begin{array}{c}\text { Stirring } \\
\text { rate }(\mathrm{rpm})\end{array}$ & $\begin{array}{c}\text { Drug } \\
\text { concentration (\%) }\end{array}$ \\
\hline F1 & $>17$ & 27 & 6000 & 1 & 50 & 400 & 30 \\
\hline $\mathrm{F} 2$ & $<17$ & 27 & 10000 & 0.5 & 50 & 600 & 30 \\
\hline F3 & $>17$ & 20 & 10000 & 0.5 & 40 & 400 & 30 \\
\hline $\mathrm{F} 4$ & $>17$ & 27 & 6000 & 0.5 & 40 & 600 & 15 \\
\hline F5 & $<17$ & 20 & 6000 & 1 & 40 & 600 & 30 \\
\hline F6 & $<17$ & 27 & 10000 & 1 & 40 & 400 & 15 \\
\hline F7 & $<17$ & 20 & 6000 & 0.5 & 50 & 400 & 15 \\
\hline F8 & $>17$ & 20 & 10000 & 1 & 50 & 600 & 15 \\
\hline
\end{tabular}

TABle 3: Particle size, zeta potential, loading efficiency, and release efficiency $\left(\mathrm{RE}_{2} \%\right)$ of different doxorubicin loaded in folate-targeted DEX/RA micelles (mean $\pm \mathrm{SD} ; n=3$ ).

\begin{tabular}{|c|c|c|c|c|c|}
\hline Formulation code & Particle size $(\mathrm{nm})$ & PDI index & Zeta potential (mv) & Loading efficiency \% & $\mathrm{RE}_{2} \%$ \\
\hline F1 & $194.4 \pm 12.9$ & $0.2 \pm 0.0$ & -1.1 & $96.1 \pm 0.9$ & $72.6 \pm 1.5$ \\
\hline F2 & $83.9 \pm 4.6$ & $0.3 \pm 0.0$ & -2.0 & $82.9 \pm 0.6$ & $49.6 \pm 2.1$ \\
\hline F3 & $140.2 \pm 29.6$ & $0.4 \pm 0.2$ & -3.5 & $92.3 \pm 1.5$ & $68.1 \pm 1.5$ \\
\hline $\mathrm{F} 4$ & $167.3 \pm 6.7$ & $0.3 \pm 0.0$ & -2.4 & $100.0 \pm 0.0$ & $67.0 \pm 1.5$ \\
\hline F5 & $128.7 \pm 6.7$ & $0.4 \pm 0.1$ & -4.8 & $100.0 \pm 0.0$ & $64.1 \pm 0.5$ \\
\hline F6 & $89.1 \pm 3.6$ & $0.5 \pm 0.0$ & -5.5 & $91.5 \pm 1.2$ & $75.3 \pm 1.0$ \\
\hline F7 & $163.0 \pm 31.2$ & $0.5 \pm 0.4$ & -3.9 & $100.0 \pm 0.0$ & $61.0 \pm 1.8$ \\
\hline F8 & $113.0 \pm 17.2$ & $0.5 \pm 0.1$ & -7.9 & $100.0 \pm 0.0$ & $58.9 \pm 2.0$ \\
\hline
\end{tabular}

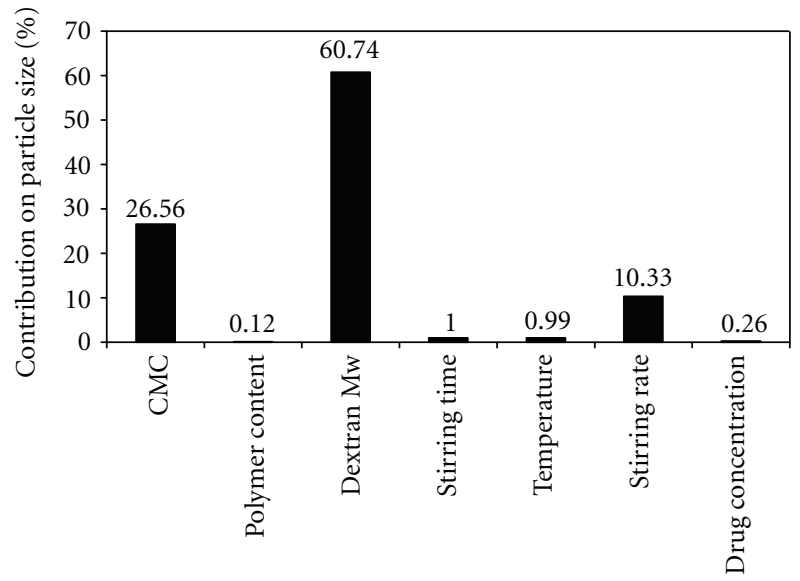

Studied parameter

FIGURE 1: Contribution percentage of different effective factors on the particle size of Dox-loaded micelles.

earlier increasing the dextran $\mathrm{Mw}$ decreased the size of micelles (Figure 1) which in turn can increase the density of surface charge of the micelles.

3.3. Loading Efficiency of Dox in the Micelles. Analysis of loading efficiency data revealed that the most effective factor on loading efficiency was DEX Mw; so that by increasing the DEX Mw (i.e., changing Mw from 6000 to 10000) the loading efficiency decreased significantly (Figure 3 ). As described

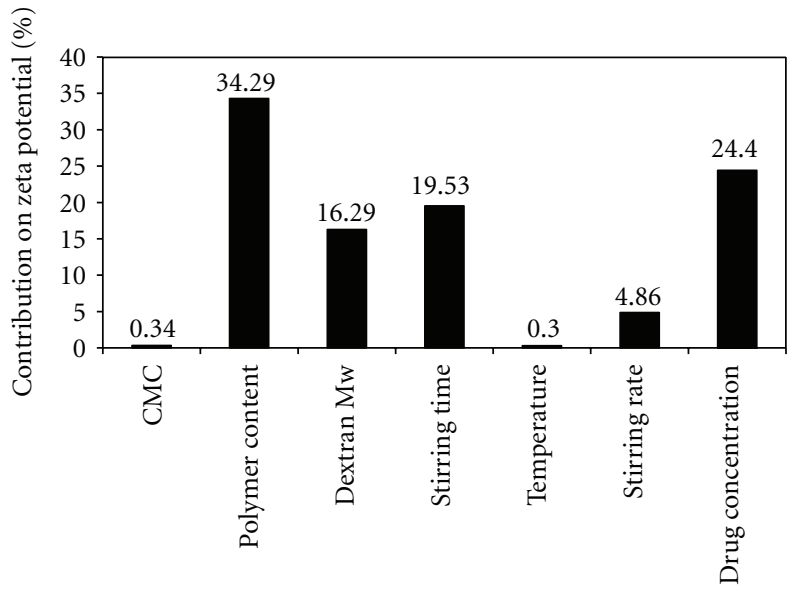

Studied parameter

FIGURE 2: Contribution percentage of different effective factors on the zeta potential of Dox-loaded micelles.

earlier increasing the Mw of DEX decreased the particle size of the micelles, and this may cause less accommodation of the drug in the micelles. Therefore, decreased drug loading efficiency by increasing DEX Mw can be explained.

As seen in Figure 3 other effective factors on loading efficiency are content of the polymer, drug concentration, and $\mathrm{CMC}$ value.

Decreasing the content of polymer (i.e., changes from level 1 to level 2) increased the loading efficiency. 


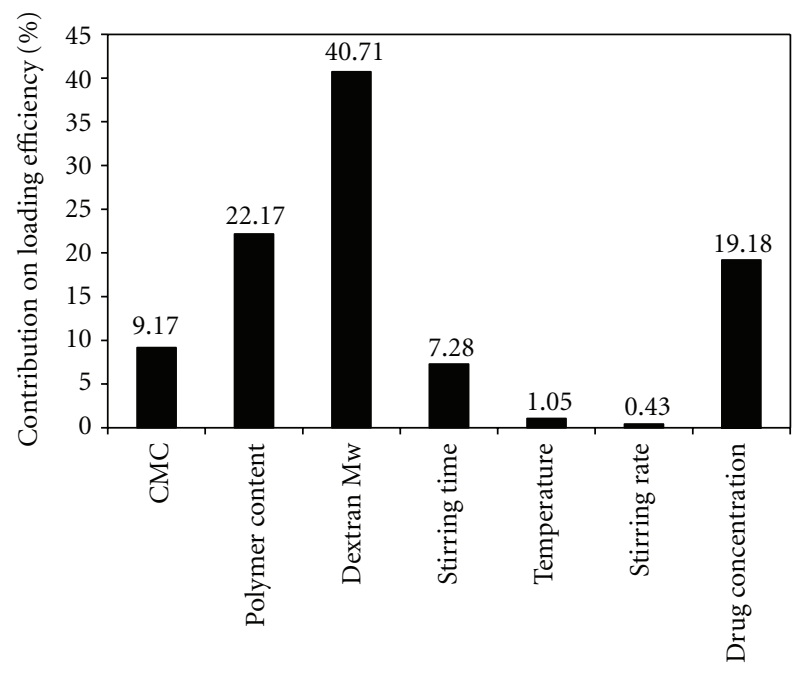

Studied parameter

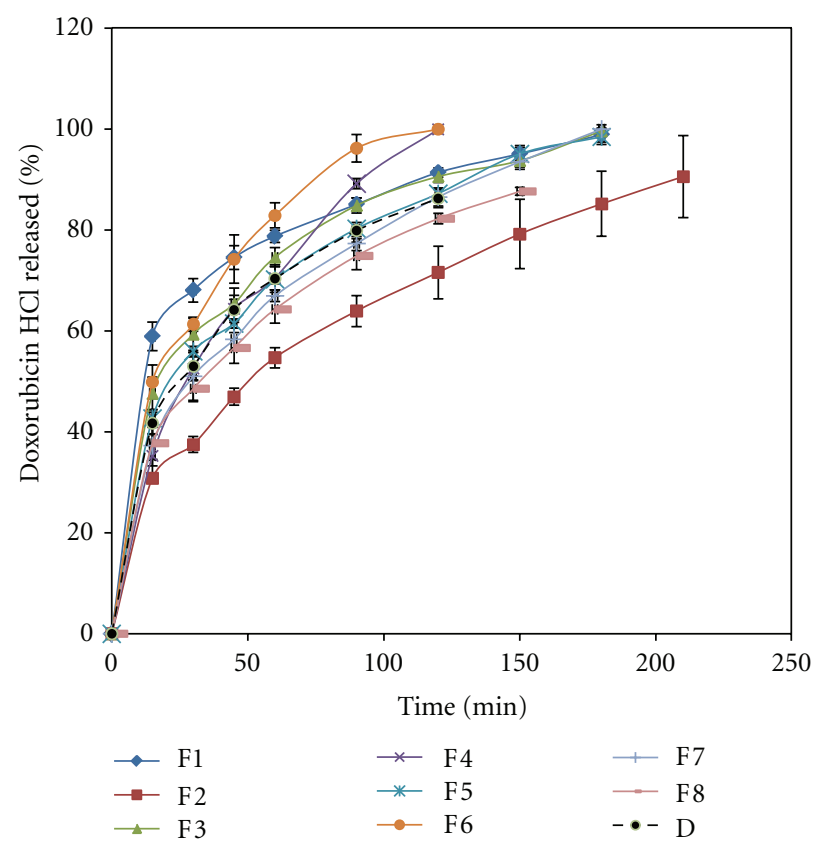

Figure 4: Release profiles of doxorubicin $\mathrm{HCl}$ from different studied formulations of FA-DEX/RA micelles.

The possible reason may be the size of the core of the micelles that cannot accommodate the loading drug in the core by increasing the drug ratio, and consequently the drug pay load will reduce.

With the same reason mentioned above decreasing the drug concentration (i.e., changing from level 1 to level 2) increased the loading efficiency in the micelles. Fattahi et al. [7] and $\mathrm{Na}$ et al. [19] demonstrated that increasing the amount of tretinoin as the feeding drug had no significant effect on loading efficiency of micelles of chitosan/retinoic acid, although drug content was significantly enhanced. This increase in drug content caused an increase in molecular hydrophobicity, which resulted in more tightly packed micelles, shrinkage of the particles, and decrease in their water content. Collectively, these effects resulted in a decrease in the size of the loaded micelles, along with an increase in the feeding amount.

Loading efficiency of micelles prepared by copolymers with $\mathrm{CMC}>17 \mu \mathrm{g} / \mathrm{mL}$ is higher than those prepared with $\mathrm{CMC}<17 \mu \mathrm{g} / \mathrm{mL}$. This may be because the loading of the ionic drug does not happen in the core of the micelles, but an electrostatic interaction causes loading of Dox in the micelles shell. The outcome of increasing the CMC values is decreasing the core size and growing the shell of the copolymer which in turn increases the ionic interaction of the drug and the micelles shell. For this reason an enhancement in loading efficiency has happened in micelles with higher $\mathrm{CMC}$ values.

3.4. In Vitro Release of Dox from Micelles. Figure 4 shows the release profiles of Dox versus time for each studied micellar formulation. This figure shows that most formulations except F4 and F6 released almost 100\% of the loaded drug during $2 \mathrm{hr}$, while others' drug release lasted for more than $4 \mathrm{hr}$. In all of them after a rapid burst release the remaining drug was released with a near zero order kinetic.
Kim et al. [20] loaded RA in the modified glycol chitosan nanoparticles and concluded the slow and prolonged release of RA from nanoparticles is attributable to the hydrophobic nature of this drug, and perhaps as well to crystallization inside the micelle's core. Here considering the hydrophilic nature of Dox $\mathrm{HCl}$ the relative fast release of this drug may be attributed to its loading in the hydrophilic shell of the micelles and also to electrostatic interaction of the drug inside the micelle's corona. Considering that the micelles could retain the drug inside the corona for a considerable time (more than $4 \mathrm{hr}$ ) due to the slow drug leakage, it can be concluded that the drug have enough time to be released when the micelles are taken up into the tumoral cells by endocytosis through their folate ligand.

Figure 5 shows that release efficiency is more affected by stirring rate during preparation of Dox-loaded micelles. As mentioned earlier by decreasing the stirring rate the particle size of the micelles increased (Figure 1). However, decreasing the stirring rate (i.e., changes level 2 to level 1) increased the release efficiency possibly due to the growing of the particle size of the micelles and decreasing the surface area of the micelles. This aggregation probably has expelled the trapped drug in the corona of the micelles, drug release was enhanced, and consequently RE was increased.

As seen in the Figure 5 the other effective factors on release efficiency were temperature and stirring time.

Decreasing the temperature in the production process of the micelles and during stirring (i.e., changing from level 1 to level 2) increased the release efficiency possibly because of the loose arrangement, less coiling of the hydrophilic DEX blocks around the core, and easier release of the entrapped drug. 
TABle 4: Comparison of the Design Expert predicted and actual values of responses studied in Dox-loaded DEX/RA micelles.

\begin{tabular}{lcccc}
\hline Response & Size $(\mathrm{nm})$ & $\begin{array}{c}\text { Zeta potential } \\
(\mathrm{mV})\end{array}$ & $\begin{array}{c}\text { Loading } \\
\text { efficiency \% }\end{array}$ & $\mathrm{RE}_{2} \%$ \\
\hline Predicted & 74.2 & -5.1 & 97.1 & 60 \\
Actual & 82.86 & -4.68 & 96.33 & 63.8 \\
Error\% & 11.67 & 8.23 & 0.79 & 6.33 \\
\hline
\end{tabular}

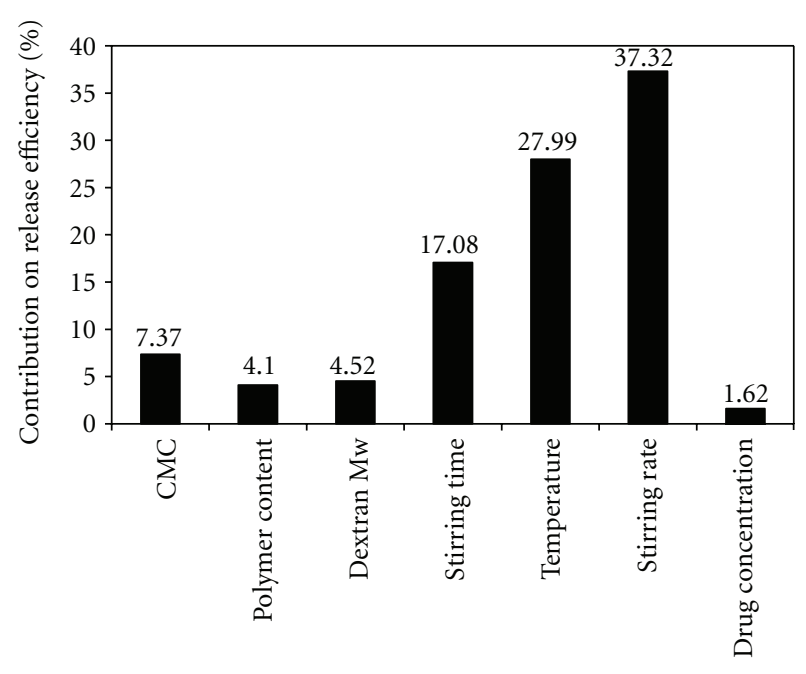

Studied parameter

FIGURE 5: Contribution percentage of different effective factors on the release efficiency of Dox from micelles.

Figure 5 also shows stirring time is another effective factor on RE of Dox from the micelles, so that increasing the stirring time during preparation of Dox-loaded micelles (i.e., changes level 2 to level 1) increased the release efficiency. Earlier in Figure 2 we showed that increasing the stirring time also increased the absolute value of zeta potential probably due to the induction of surface charge in micellar nanoparticles. This in turn increased the loading efficiency of the drug in micelles by enhanced electrostatic interactions. Possibly greater loading efficiency has caused greater drug release and $\mathrm{RE}$.

3.5. Optimization of the Production Process of Dox-Loaded Micelles. Computer optimization process by Design Expert software (version 7.2, US) and a desirability function determined the effect of the levels of independent variables on the responses. All responses were fitted to the linear model. The constraint of particle size was $83.9 \leq Y_{1} \leq 194.4 \mathrm{~nm}$ with targeting the particle size on minimum, for zeta potential it was $-1.07 \leq Y_{2} \leq-7.95 \mathrm{mV}$ while the target was set in range of zeta potential values, for loading efficiency the constraint was $82.9 \leq Y_{3} \leq 100 \%$ with the goal set at the range of obtained data (Table 3 ), and the $\mathrm{RE}_{2} \%$ constraint was $49.6 \leq$ $Y_{4} \leq 75.27 \%$ with the target set at the range of the obtained data of Table 3. Considering the data of Table 3 optimization was carried out by Design Expert software, and optimized micelles processing situation was suggested by desirability of $100 \%$ as using copolymers with CMC $<17 \mu \mathrm{g} / \mathrm{mL}, 20.1 \mathrm{mg}$ copolymer content, DEX molecular weight of 10000, drug concentration of $15.9 \%$, temperature of $40.7^{\circ} \mathrm{C}$, and $1 \mathrm{hr}$ stirring at a rate of $560 \mathrm{rpm}$. The predicted and actual values of responses are shown in Table 4.

As this Table shows the actual results were in close accordance with the predicted values by the software.

\section{Conclusions}

Processing variables for formation of FA-DEX/RA micelles can be optimized by a standard orthogonal $\mathrm{L}_{8}$ array using a Taguchi design. The size of FA-DEX/RA micelles is more affected by DEX Mw, the CMC of the copolymer, and stirring rate while their zeta potential and drug loading efficiency are more affected by the copolymer and drug content and DEX Mw. Stirring time during preparation of the micelles has also significant effect on zeta potential. Stirring rate and time and also the temperature of the solution are the most effective variable factors on the Dox release efficiency from the micelles.

\section{Acknowledgments}

The authors wish to thank the Vice Chancellery of Isfahan University of Medical Sciences that supported this work. This paper is extracted from the dissertation of Mohammad Nayebsadrian the PharmD Student of Faculty of Pharmacy, Isfahan University of Medical Sciences.

\section{References}

[1] K. Kataoka, A. Harada, and Y. Nagasaki, "Block copolymer micelles for drug delivery: design, characterization and biological significance," Advanced Drug Delivery Reviews, vol. 47, no. 1, pp. 113-131, 2001.

[2] U. Kedar, P. Phutane, S. Shidhaye, and V. Kadam, "Advances in polymeric micelles for drug delivery and tumor targeting," Nanomedicine, vol. 6, no. 6, pp. 714-729, 2010.

[3] H. S. Yoo and T. G. Park, "Folate receptor targeted biodegradable polymeric doxorubicin micelles," Journal of Controlled Release, vol. 96, no. 2, pp. 273-283, 2004.

[4] V. P. Torchilin, "Structure and design of polymeric surfactantbased drug delivery systems," Journal of Controlled Release, vol. 73, no. 2-3, pp. 137-172, 2001.

[5] A. Aumelas, A. Serrero, A. Durand, E. Dellacherie, and M. Leonard, "Nanoparticles of hydrophobically modified dextrans as potential drug carrier systems," Colloids and Surfaces $B$, vol. 59, no. 1, pp. 74-80, 2007.

[6] R. Mehvar, "Dextrans for targeted and sustained delivery of therapeutic and imaging agents," Journal of Controlled Release, vol. 69, no. 1, pp. 1-25, 2000.

[7] A. Fattahi, M.-A. Golozar, J. Varshosaz, H. Mirmohammad Sadeghi, and M. Fathi, "Preparation and characterization of micelles of oligomeric chitosan linked to all-trans retinoic acid," Carbohydrate Polymers, vol. 87, no. 2, pp. 1176-1184, 2012.

[8] C. Díaz, E. Vargas, and O. Gätjens-Boniche, "Cytotoxic effect induced by retinoic acid loaded into galactosyl-sphingosine containing liposomes on human hepatoma cell lines," International Journal of Pharmaceutics, vol. 325, no. 1-2, pp. 108-115, 2006. 
[9] R. J. Li, X. Ying, Y. Zhang et al., "All-trans retinoic acid stealth liposomes prevent the relapse of breast cancer arising from the cancer stem cells," Journal of Controlled Release, vol. 149, no. 3, pp. 281-291, 2011.

[10] H. Li, Y. Lu, L. Piao et al., "Targeting human clonogenic acute myelogenous leukemia cells via folate conjugated liposomes combined with receptor modulation by all-trans retinoic acid," International Journal of Pharmaceutics, vol. 402, no. 12, pp. 57-63, 2010.

[11] F. Q. Hu, X. L. Wu, Y. Z. Du, J. You, and H. Yuan, "Cellular uptake and cytotoxicity of shell crosslinked stearic acid-grafted chitosan oligosaccharide micelles encapsulating doxorubicin," European Journal of Pharmaceutics and Biopharmaceutics, vol. 69, no. 1, pp. 117-125, 2008.

[12] X. Q. Pan, X. Zheng, G. Shi, H. Wang, M. Ratnam, and R. J. Lee, "Strategy for the treatment of acute myelogenous leukemia based on folate receptor $\beta$-targeted liposomal doxorubicin combined with receptor induction using all-trans retinoic acid," Blood, vol. 100, no. 2, pp. 594-602, 2002.

[13] H. Zhao and L. Y. L. Yung, "Selectivity of folate conjugated polymer micelles against different tumor cells," International Journal of Pharmaceutics, vol. 349, no. 1-2, pp. 256-268, 2008.

[14] J. Varshosaz, F. Hassanzadeh, H. Sadeghi, M. Nayebsadrian, M. Banitalebi, and M. Rostami, "Synthesis and characterization of folate-targeted dextran/retinoic acid micelles for doxorubicin delivery in acute leukemia," Submitted. In press.

[15] S. R. Croy and G. S. Kwon, "Polymeric micelles for drug delivery," Current Pharmaceutical Design, vol. 12, no. 36, pp. 46694684, 2006.

[16] W. Y. Fowlkes and C. M. Creveling, Engineering Methods for Robust Product Design Using Taguchi Methods in Technology and Product Development, Addison-Wesley, New York, NY, USA, 1995.

[17] R. K. Averineni, G. V. Shavi, A. K. Gurram et al., "PLGA 50:50 nanoparticles of paclitaxel: development, in vitro anti-tumor activity in BT-549 cells and in vivo evaluation," Bulletin of Material Science, vol. 35, no. 3, pp. 319-326, 2012.

[18] N. Mohammadpour Dounighi, R. Eskandari, M. R. Avadi, H. Zolfagharian, A. Mir Mohammad Sadeghi, and M. Rezayat, "Preparation and in vitro characterization of chitosan nanoparticles containing Mesobuthus eupeus scorpion venom as an antigen delivery system," Journal of Venomous Animals and Toxins Including Tropical Diseases, vol. 18, no. 1, pp. 44-52, 2012.

[19] K. Na, K. H. Park, S. W. Kim, and Y. H. Bae, "Self-assembled hydrogel nanoparticles from curdlan derivatives: characterization, anti-cancer drug release and interaction with a hepatoma cell line (HepG2)," Journal of Controlled Release, vol. 69, no. 2, pp. 225-236, 2000.

[20] J. H. Kim, Y. S. Kim, S. Kim et al., "Hydrophobically modified glycol chitosan nanoparticles as carriers for paclitaxel," Journal of Controlled Release, vol. 111, no. 1-2, pp. 228-234, 2006. 

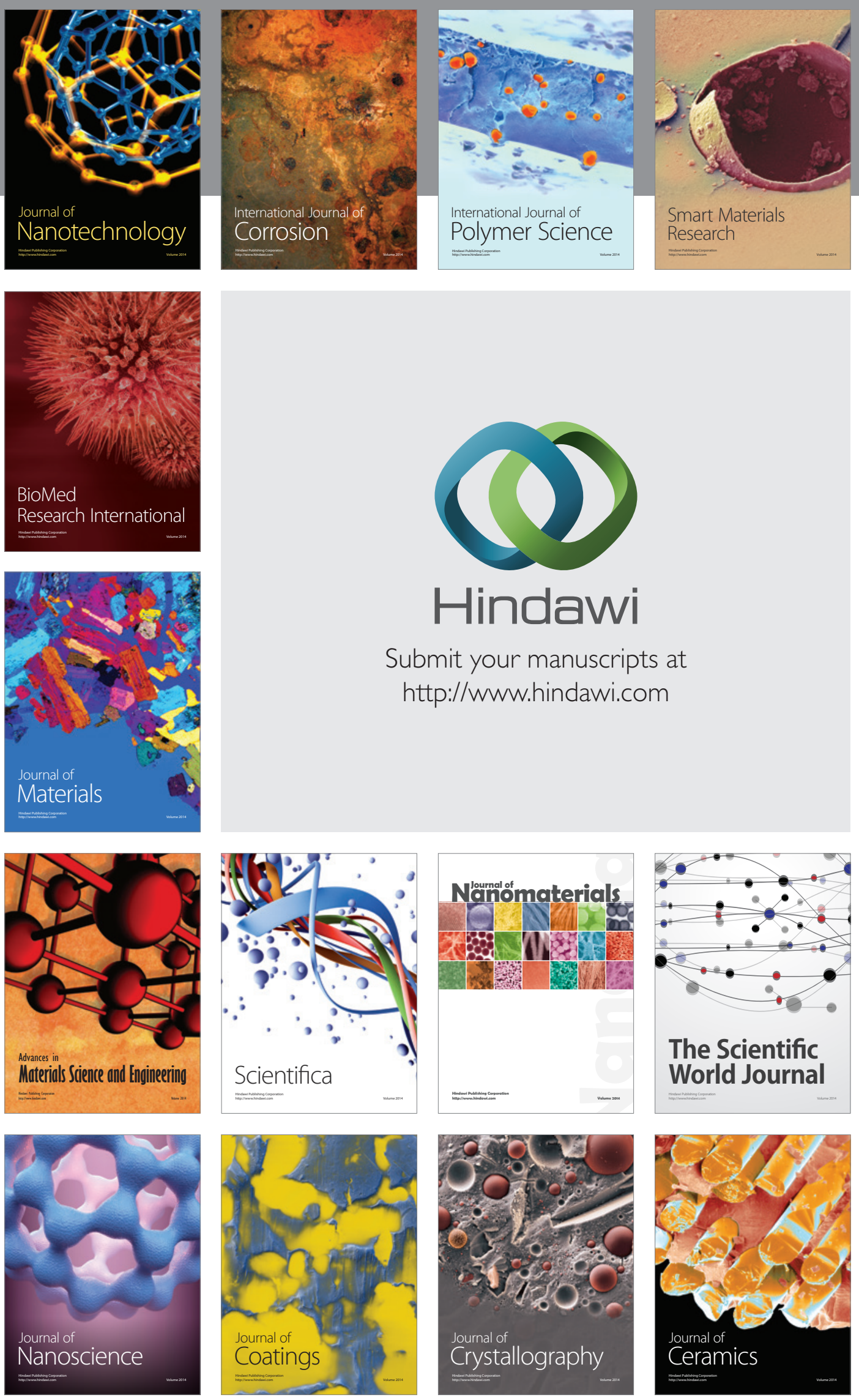

The Scientific World Journal

Submit your manuscripts at

http://www.hindawi.com

\section{World Journal}

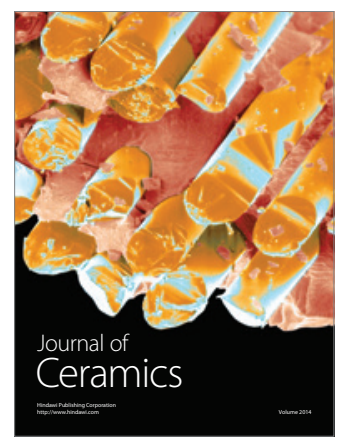

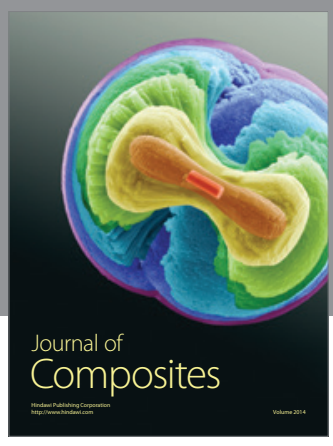
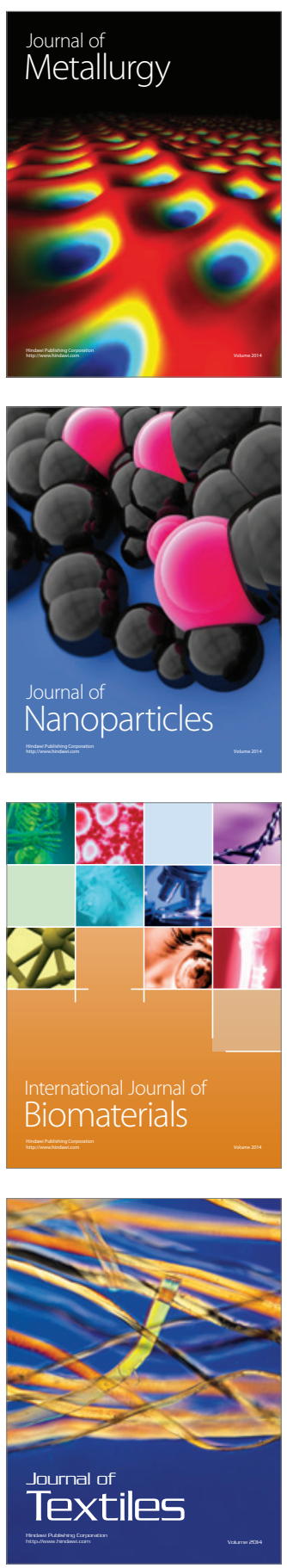